\title{
$\gamma$-tocotrienol attenuates TNF- $\alpha$-induced changes in secretion and gene expression of MCP-1, IL-6 and adiponectin in 3T3-L1 adipocytes
}

\author{
TETSURO MATSUNAGA ${ }^{1 *}$, AYAE SHOJI ${ }^{2 *}$, NING GU ${ }^{3}$, ERINA JOO $^{2,4}$, SHIHO LI $^{2}$, TETSUYA ADACHI $^{5}$, \\ HANAE YAMAZAKI ${ }^{1}$, KOICHIRO YASUDA $^{6}$, TAKASHI KONDOH ${ }^{1}$ and KINSUKE TSUDA ${ }^{2}$ \\ ${ }^{1}$ Ajinomoto Integrative Research for Advanced Dieting, Graduate School of Agriculture; ${ }^{2}$ Laboratory of Metabolism, \\ Graduate School of Human and Environmental Studies, Kyoto University, Kyoto, Japan; ${ }^{3}$ Department of Life Science \\ and Engineering, Harbin Institute of Technology, Harbin, P.R. China; ${ }^{4}$ Department of Diabetes and Clinical Nutrition, \\ Graduate School of Medicine, Kyoto University; ${ }^{5}$ Department of Pathology and Cell Regulation, \\ Kyoto Prefectural University of Medicine, Kyoto; ${ }^{6}$ Saiseikai-Noe Hospital, Osaka, Japan
}

Received August 23, 2011; Accepted December 22, 2011

DOI: $10.3892 / \mathrm{mmr} .2012 .770$

\begin{abstract}
Tocotrienols, members of the vitamin E family, have been shown to possess anti-inflammatory properties and display activity against a variety of chronic diseases, such as cancer, cardiovascular and neurological diseases. However, whether tocotrienols contribute to the prevention of inflammatory responses in adipose tissue remains to be elucidated. In this study, we examined the effects of $\gamma$-tocotrienol, the most common tocotrienol isomer, on tumor necrosis factor- $\alpha$ (TNF- $\alpha$ )-induced inflammatory responses by measuring the expression of the adipokines, monocyte chemoattractant protein-1 (MCP-1), interleukin-6 (IL-6) and adiponectin in 3T3-L1 adipocytes. Exposure to TNF- $\alpha(10 \mathrm{ng} / \mathrm{ml})$ for $24 \mathrm{~h}$ increased MCP-1 and IL-6 secretion, and decreased adiponectin secretion and peroxisome proliferator-activated receptor- $\gamma(P P A R \gamma)$ mRNA expression. $\gamma$-tocotrienol effectively improved the TNF- $\alpha$-induced adverse changes in MCP-1, IL-6 and adiponectin secretion, and in $M C P-1, I L-6$, adiponectin and $P P A R \gamma$ mRNA expression. Furthermore, TNF- $\alpha$-mediated I $\kappa$ B- $\alpha$ phosphorylation and nuclear factor- $\kappa \mathrm{B}$ $(\mathrm{NF}-\kappa \mathrm{B})$ activation were significantly suppressed by the $\gamma$-tocotrienol treatment. Our results suggest that $\gamma$-tocotrienol may improve obesity-related functional abnormalities in adipocytes by attenuating $\mathrm{NF}-\kappa \mathrm{B}$ activation and the expression of inflammatory adipokines.
\end{abstract}

Correspondence to: Dr Kinsuke Tsuda, Laboratory of Metabolism, Graduate School of Human and Environmental Studies, Kyoto University, Yoshida, Nihonmatsu-cho, Sakyo-ku, Kyoto 606-8501, Japan

E-mail: dmmed-028@umin.ac.jp

*Contributed equally

Key words: tocotrienol, vitamin E, adipocyte, adipokine, inflammation

\section{Introduction}

Obesity is closely associated with a low-grade state of inflammation, resulting from enlargement of adipocytes and increased macrophage infiltration into the adipose tissue $(1,2)$. Obese adipose tissue is characterized by abnormal production and secretion of adipokines as well as activation of inflammatory signaling in adipocytes $(1,2)$. As obesity-induced inflammation in adipocytes develops, secretion of pro-inflammatory adipokines, including monocyte chemoattractant protein-1 (MCP-1) and interleukin-6 (IL-6), increases, which in turn leads to a decrease in insulin sensitivity $(1,2)$. Furthermore, it has been suggested that the nuclear factor- $\kappa \mathrm{B}(\mathrm{NF}-\kappa \mathrm{B})$ pathway plays an important role in facilitating adipocyte inflammation. The $\mathrm{NF}-\kappa \mathrm{B}$ signal is related to the up-regulation of pro-inflammatory adipokines and the down-regulation of adiponectin which has anti-inflammatory and insulin-sensitizing properties (3-5).

Tumor necrosis factor- $\alpha(\mathrm{TNF}-\alpha)$ is one of the most important molecules in obesity. TNF- $\alpha$ released from adipose tissue and macrophages is markedly increased in obese or diabetic subjects $(6,7)$. It induces insulin resistance by modulating the secretion of pro-inflammatory adipokines, such as MCP-1 and IL-6, and directly contributing to the inhibition of adiponectin production (6-9). It has been shown that TNF- $\alpha$ activates the $\mathrm{NF}-\kappa \mathrm{B}$ pathway and suppresses the expression of peroxisome proliferator-activated receptor- $\gamma(\operatorname{PPAR} \gamma)$, which is a strong transcriptional inducer of adiponectin (10).

Vitamin E exists in nature as eight vitamers that are subdivided into two subgroups called tocopherols and tocotrienols, each including the $\alpha$-, $\beta$-, $\gamma$ - and $\delta$-forms $(11,12)$. Tocopherols possess a saturated phytyl chain, whereas tocotrienols contain an unsaturated side chain. Vitamin E isomers differ from each other by the number of methyl groups in the chroman ring. Although tocopherols and tocotrienols exhibit strong antioxidant activities $(11,12)$, most vitamin E studies have focused on tocopherols, and very little is known about tocotrienols. Tocotrienols are primarily found in oat, wheat germ, rice bran and palm oil (11). However, previous studies have found that 
tocotrienols have various physiological activities, including anticancer, cardiovascular-protective, hypocholesterolemic and neuroprotective activities $(12,13)$. Among the tocotrienol isomers, $\gamma$-tocotrienol, the most common tocotrienol isomer, has been well documented for its physiological availability. It has been shown that $\gamma$-tocotrienol suppresses adipocyte differentiation (14), and oral administration of $\gamma$-tocotrienol significantly decreases body fat in rats (15).

Tocotrienols have been shown to possess anti-inflammatory effects in certain cell types, which are mediated by inhibition of the NF- $\kappa \mathrm{B}$ activation pathway $(16,17)$. It remains unknown whether $\gamma$-tocotrienol exerts such effects in adipocytes. Although administered $\gamma$-tocotrienol can be accumulated in adipose tissue (18), there have been no reports on its effects on adipokine regulation. Therefore, in the present study, we examined the effects of $\gamma$-tocotrienol on the TNF- $\alpha$-induced changes in secretion and gene expression of inflammatoryrelated adipokines, and activation of the $\mathrm{NF}-\kappa \mathrm{B}$ pathway in 3T3-L1 adipocytes.

\section{Materials and methods}

Reagents. Recombinant TNF- $\alpha$ was purchased from R\&D Systems (Minneapolis, MN, USA). $\gamma$-tocotrienol was from Cayman Chemical (Ann Arbor, MI, USA). Dulbecco's modified Eagle's medium (DMEM) and insulin were from Sigma-Aldrich (St. Louis, MO, USA). Isobutylmethylxanthine (IBMX), sodium pyruvate and dexamethasone (DEX) were from Nacalai Tesque (Kyoto, Japan). Fetal bovine serum (FBS) was from Gibco-BRL (Rockville, MD, USA).

Cell culture and treatment. 3T3-L1 cells (Health Science Research Resources Bank, Osaka, Japan), were maintained in DMEM containing $25 \mathrm{mM}$ glucose, $1 \mathrm{mM}$ sodium pyruvate and $10 \% \mathrm{FBS}$, in a $5 \% \mathrm{CO}_{2}$ atmosphere at $37^{\circ} \mathrm{C}$. Differentiation was induced by replacing the medium with FBS-supplemented DMEM containing $200 \mathrm{nM}$ insulin, $0.5 \mathrm{mM}$ IBMX and $1 \mu \mathrm{M}$ DEX for 2 days. After another 2 days of incubation in 10\% FBS/DMEM medium with $200 \mathrm{nM}$ insulin, the medium was replaced every 2 days with $10 \% \mathrm{FBS} /$ DMEM medium until $>90 \%$ of cells were demonstrating the adipocyte phenotype. On days 6-8 of differentiation, 3T3-L1 adipocytes were pre-treated with $0.024-2.4 \mu \mathrm{M} \gamma$-tocotrienol for $6 \mathrm{~h}$ and then stimulated for $24 \mathrm{~h}$ with $10 \mathrm{ng} / \mathrm{ml} \mathrm{TNF}-\alpha$. Following the 24-h incubation, the conditioned medium was collected for measurement of adipokines by ELISA. Total RNA and protein were isolated and the expression levels of the genes and proteins of interest were evaluated by quantitative real-time RT-PCR and Western blotting, respectively.

Real-time RT-PCR analysis. Total RNA was extracted from 3T3-L1 adipocytes using Sepasol-RNA I (Nacalai Tesque, Kyoto, Japan). For complementary DNA synthesis, $1 \mu \mathrm{g}$ of total RNA was reverse-transcribed using the reverse transcription system (PrimeScript RT reagent kit, Takara Bio, Shiga, Japan). Real-time RT-PCR was performed by the ABI Prism 7000 sequence detection system (Applied Biosystems, Foster City, CA, USA) using SYBR Green fluorescence signals (SYBR Premix Ex Taq II; Takara Bio). The following oligonucleotide primer pairs were used: mouse $M C P-1,5^{\prime}-\mathrm{CCA}$
CTC ACC TGC TGC TAC TCA T-3' (forward) and 5'-TGG TGA TCC TCT TGT AGC TCT CC-3' (reverse); mouse $I L-6$, 5'-GCT ACC AAA CTG GAT ATA ATC AGG A-3' (forward) and 5'-CCA GGT AGC TAT GGT ACT CCG AA-3' (reverse); mouse adiponectin, 5'-GTT CTA CTG CAA CAT TCC GG-3' (forward) and 5'-TAC ACC TGG AGC CAG ACT TG-3' (reverse); mouse $P P A R \gamma$, 5'-GGC GAT CTT GAC AGG AAA GAC-3' (forward) and 5'-CCC TTG AAA AAT TCG GAT GG-3' (reverse); mouse 36B4, 5'-CCG GAT GTG AGG CAG CAG-3' (forward) and 5'-GCT CCA AGC AGA TGC AGC A-3' (reverse). Expression levels of RNA, expressed as relative mRNA levels compared to control, were calculated after normalization to $36 B 4$.

Adipokine ELISA. The culture medium from 3T3-L1 adipocytes was collected from each sample $24 \mathrm{~h}$ after TNF- $\alpha$ treatment. The concentrations of MCP-1, IL- 6 and adiponectin were assayed using a mouse MCP-1 ELISA kit (R\&D systems), a mouse IL-6 ELISA kit (R\&D systems) and a mouse adiponectin ELISA kit (R\&D systems).

Protein isolation and Western blotting. Whole cell lysates were prepared using RIPA buffer (50 mM Tris-HCl, $\mathrm{pH} 8.0$, $150 \mathrm{mM} \mathrm{NaCl}, 1 \% \mathrm{NP}-40,0.5 \% \mathrm{Na}$-deoxycholate and $0.1 \%$ SDS) containing a protease inhibitor cocktail (Nacalai Tesque) and complete phosphatase inhibitors (Nacalai Tesque). Lysates were centrifuged at $12,000 \mathrm{~g}$ for $10 \mathrm{~min}$ at $4^{\circ} \mathrm{C}$ and the supernatants were boiled in SDS loading buffer. The boiled samples were separated by an SDS-PAGE gradient gel (10-20\%) and transferred to PVDF membranes (Bio-Rad Laboratories, CA, USA). Membranes were blocked with blocking reagent (Blocking One-P; Nacalai Tesque) and incubated with anti-

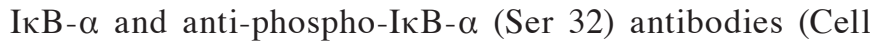
Signaling Technology, Beverly, MA, USA), followed by incubation with horseradish peroxidase-conjugated secondary antibodies. Western blot analysis was conducted using an enhanced chemiluminescence detection system (ECL-Plus; Amersham Pharmacia, Arlington, IL, USA).

Quantification of $N F-\kappa B$ activation. To quantify NF- $\kappa$ B activity, nuclear extracts were prepared using Nuclear Extract kit (Active Motif, Carlsbad, CA, USA) and analyzed with a sensitive ELISA-based kit (PathScan Total NF- $\kappa$ B p65 Sandwich ELISA kit; Cell Signaling Technology) to quantify $\mathrm{NF}-\kappa \mathrm{B}$ activity, according to the manufacturer's instructions.

Statistical analysis. Results are expressed as the means \pm SEM. Data were analyzed using one-way analysis of variance (ANOVA) between groups with the Dunnett post-hoc test. Statistical analyses were performed using the SPSS 11.0 software (SPSS Inc., Chicago, IL, USA). Significant differences were considered to be present at $\mathrm{P}<0.05$.

\section{Results}

Effects of $\gamma$-tocotrienol on adipokine secretion in TNF- $\alpha$ treated 3T3-L1 adipocytes. To investigate whether $\gamma$-tocotrienol affects the TNF- $\alpha$-induced secretion of adipokines, 3T3-L1 adipocytes were pre-treated with various concentrations of $\gamma$-tocotrienol for $6 \mathrm{~h}$ and then incubated with $10 \mathrm{ng} / \mathrm{ml} \mathrm{TNF}-\alpha$ 
A

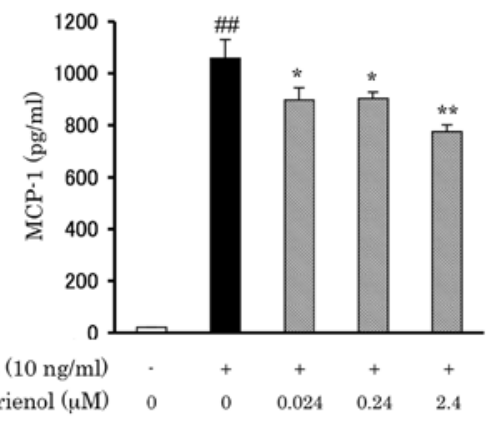

B

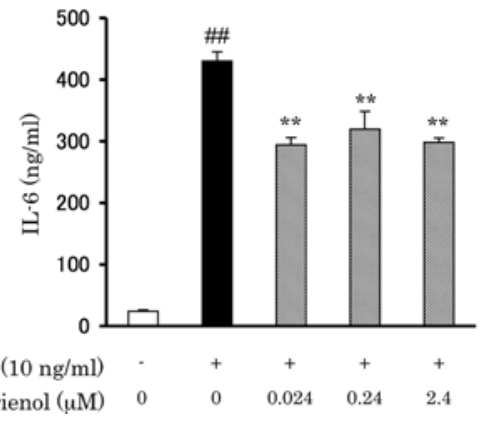

C

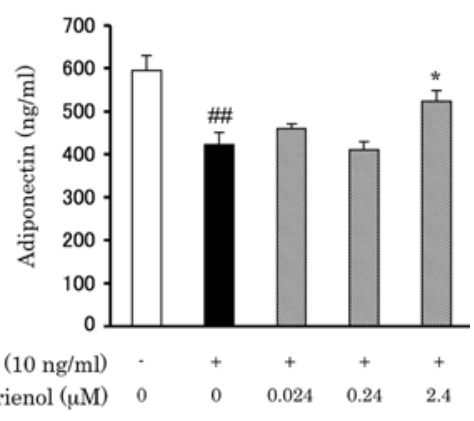

Figure 1. Effects of $\gamma$-tocotrienol on the TNF- $\alpha$-induced changes in adipokine secretion. 3T3-L1 adipocytes were pre-treated with various concentrations of $\gamma$-tocotrienol $(0.024-2.4 \mu \mathrm{M})$ for $6 \mathrm{~h}$ and then exposed to $10 \mathrm{ng} / \mathrm{ml} \mathrm{TNF}-\alpha$ for $24 \mathrm{~h}$. The levels of (A) MCP-1, (B) IL-6 and (C) adiponectin in the culture medium were measured by the ELISA assay. Data are expressed as the means $\pm \mathrm{SEM}(\mathrm{n}=5)$. ${ }^{\# \#} \mathrm{P}<0.01$ vs. the untreated group; ${ }^{*} \mathrm{P}<0.05$, ${ }^{* *} \mathrm{P}<0.01 \mathrm{vs}$. the $\mathrm{TNF}-\alpha$ only-treated group.

A

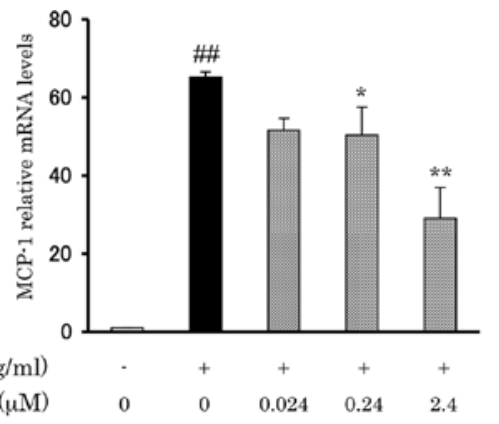

C

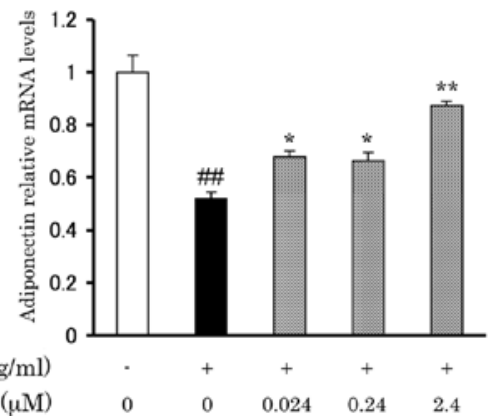

B

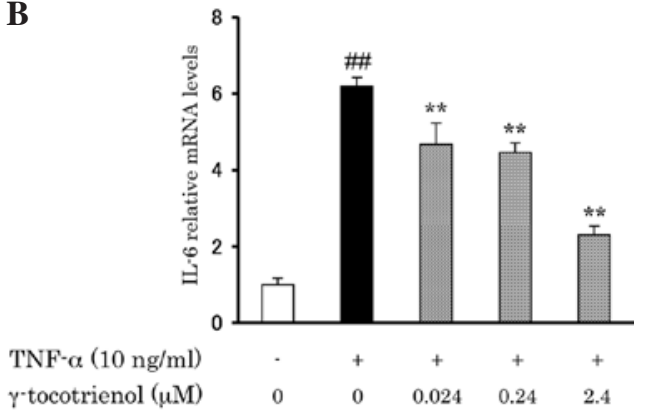

D

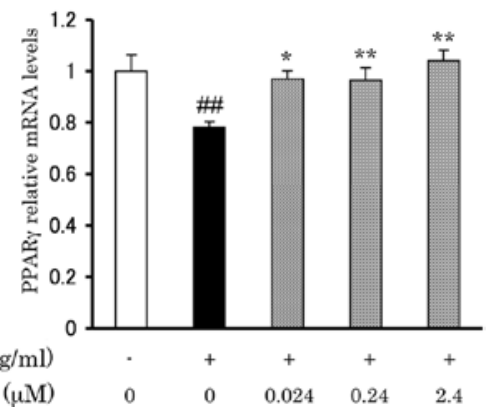

Figure 2. Effects of $\gamma$-tocotrienol on the TNF- $\alpha$-induced changes in mRNA expression of adipokines and PPAR $\gamma$. 3T3-L1 adipocytes were pre-treated with various concentrations of $\gamma$-tocotrienol (0.024-2.4 $\mu \mathrm{M})$ for $6 \mathrm{~h}$ and then exposed to $10 \mathrm{ng} / \mathrm{ml} \mathrm{TNF}-\alpha$ for $24 \mathrm{~h}$. Total RNA was extracted for measuring the mRNA expression levels of (A) MCP-1, (B) IL-6 (C) adiponectin and (D) PPAR $\gamma$ by real-time RT-PCR. Values were normalized to $36 B 4$ mRNA levels and expressed relative to the untreated group. Data are expressed as the means \pm SEM $(\mathrm{n}=6)$. ${ }^{\# \#} \mathrm{P}<0.01$ vs. the untreated group; ${ }^{*} \mathrm{P}<0.05,{ }^{* *} \mathrm{P}<0.01 \mathrm{vs}$. the TNF- $\alpha$ only-treated group.

for $24 \mathrm{~h}$. Adipokines secreted into the conditioned medium were measured by an ELISA assay. TNF- $\alpha$-induced increases in MCP-1 and IL-6 secretion were significantly inhibited by $\gamma$-tocotrienol treatment (Fig. 1A and B). At the $\gamma$-tocotrienol concentration of $2.4 \mu \mathrm{M}$, the secretions of MCP-1 and IL-6 were decreased by 27.7 and $36.5 \%$, respectively. By contrast, adiponectin secretion, which was decreased by TNF- $\alpha$ stimulation, was restored by $\gamma$-tocotrienol treatment (Fig. 1C). In the presence of $2.4 \mu \mathrm{M} \gamma$-tocotrienol, adiponectin levels were 1.24-fold higher than with TNF- $\alpha$ alone. Thus, treatment with $\gamma$-tocotrienol attenuated the effects of TNF- $\alpha$ on the secretions of three adipokines.

Effects of $\gamma$-tocotrienol on adipokine gene expression in TNF- $\alpha$-treated 3T3-L1 adipocytes. The gene expression of
MCP-1, IL-6 and adiponectin tested by real-time quantitative RT-PCR analysis is shown in Fig. 2. The enhanced expression of $M C P-1$ and $I L-6$ mRNA by TNF- $\alpha$-stimulation was effectively inhibited by $\gamma$-tocotrienol treatment (Fig. 2A and B). At $2.4 \mu \mathrm{M} \gamma$-tocotrienol, the gene expression of $M C P-1$ and $I L-6$ was suppressed by 55.6 and $62.8 \%$, respectively. $\gamma$-tocotrienol also attenuated the inhibiting effect of TNF- $\alpha$ on adiponectin gene expression (Fig. 2C). The expression of adiponectin mRNA was restored to $87.2 \%$ of control by $\gamma$-tocotrienol $(2.4 \mu \mathrm{M})$ treatment. Furthermore, PPAR $\gamma$ mRNA expression, which was suppressed by TNF- $\alpha$, was restored to the control level by treatment with $\gamma$-tocotrienol at all concentrations tested (Fig. 2D). Thus, TNF- $\alpha$-induced changes in the mRNA transcription levels of adipokines were also effectively suppressed by $\gamma$-tocotrienol. 

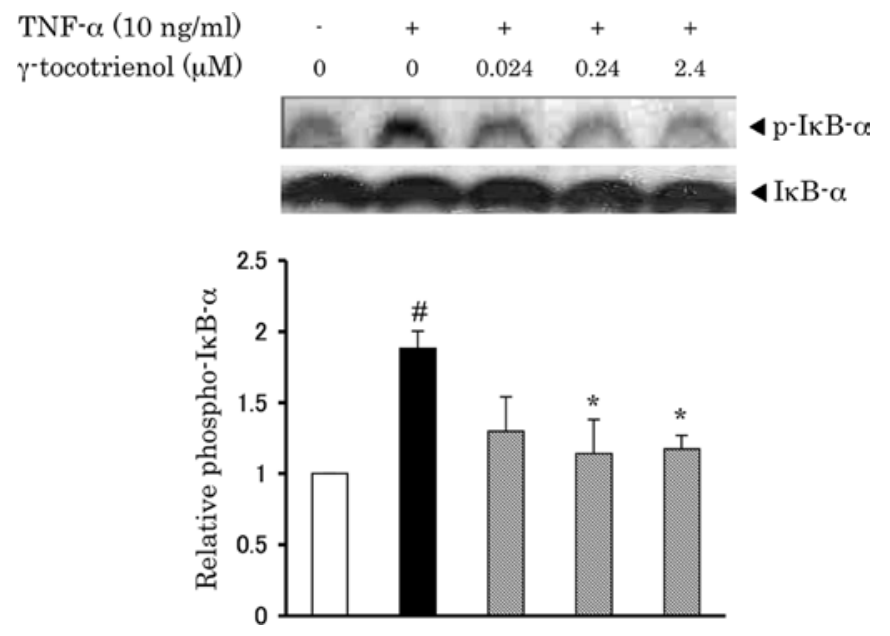

Figure 3. $\gamma$-tocotrienol inhibits IкB- $\alpha$ phosphorylation in TNF- $\alpha$-treated 3T3-L1 adipocytes. 3T3-L1 adipocytes were pre-treated with various concentrations of $\gamma$-tocotrienol $(0.024-2.4 \mu \mathrm{M})$ for $6 \mathrm{~h}$ and then exposed to $10 \mathrm{ng} /$ $\mathrm{ml}$ TNF- $\alpha$ for $24 \mathrm{~h}$. Phosphorylation of IKB- $\alpha$ was evaluated by Western blotting. Values are expressed as fold-increase compared to the untreated group. Data are expressed as the means \pm SEM $(n=3) .{ }^{~} \mathrm{P}<0.05$ vs. the untreated group; ${ }^{*} \mathrm{P}<0.05$ vs. the TNF- $\alpha$ only-treated group.

$\gamma$-tocotrienol inhibits TNF- $\alpha$-induced activation of $N F-\kappa B$ in 3T3-L1 adipocytes. Activation of transcription factor $\mathrm{NF}-\kappa \mathrm{B}$ plays an important role in the TNF- $\alpha$-mediated inflammation progress, the down-regulation of adiponectin and the up-regulation of inflammatory molecules, including MCP-1 and IL-6 (3-5). The release and nuclear translocation of active NF- $\kappa \mathrm{B}$ are regulated by phosphorylation of I $\mathrm{B}-\alpha(19)$. To further evaluate whether the anti-inflammatory function of $\gamma$-tocotrienol is mediated by $\mathrm{NF}-\kappa \mathrm{B}$, the effects of $\gamma$-tocotrienol on I $\kappa \mathrm{B}-\alpha$ phosphorylation and NF- $\mathrm{B}$ (p65) nuclear translocation were examined by Western blot analysis and ELISA assay. As shown in Fig. 3, TNF- $\alpha$ increased the phosphorylation level of I $\mathrm{B}-\alpha$, which was attenuated by treatment with $\gamma$-tocotrienol. Furthermore, $\gamma$-tocotrienol effectively suppressed the TNF- $\alpha$ -

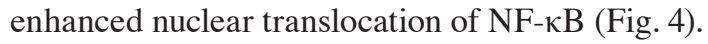

\section{Discussion}

Obesity is considered to be a state of low-grade inflammation in adipose tissues, which is closely associated with the development of insulin resistance $(1,2)$. This inflammatory condition is partly caused by macrophage infiltration into the adipose tissue and, subsequently, the inflamed adipocyte itself enhances the production of various pro-inflammatory cytokines, including TNF- $\alpha$, MCP-1 and IL-6 $(1,2)$. It has been reported that TNF- $\alpha$ levels are increased in obese subjects, and that they induce the inflammation of adipocytes through the elevation of inflammatory adipokines $(6,7)$. Furthermore, it is known that TNF- $\alpha$ is one of the negative regulators of adiponectin, attenuating its beneficial effects, such as anti-inflammation and facilitation of insulin sensitivity $(8,9)$. Therefore, anti-inflammatory treatment could be an effective way to prevent or treat insulin resistance and type 2 diabetes. Several reports have suggested that dietary tocotrienols accumulate in adipose tissue and skin (18); however, not much is known about their physiological effects on adipocytes. It has also been reported that treatment

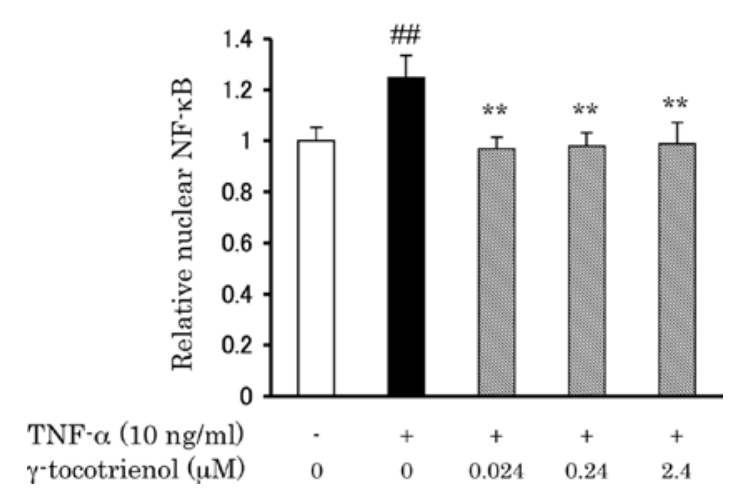

Figure 4. Effect of $\gamma$-tocotrienol on NF- $\kappa$ B activity in TNF- $\alpha$-treated 3T3-L1 adipocytes. 3T3-L1 adipocytes were pre-treated with various concentrations of $\gamma$-tocotrienol $(0.024-2.4 \mu \mathrm{M})$ for $6 \mathrm{~h}$ and then exposed to $10 \mathrm{ng} / \mathrm{ml}$ TNF- $\alpha$ for $24 \mathrm{~h}$. The nuclear fraction was obtained and NF- $\mathrm{B}$ (p65) levels were assayed by ELISA, as described in Materials and methods. Values are expressed relative to the untreated group. Data are expressed as the means \pm SEM ( $=5) .{ }^{\#} \mathrm{P}<0.01$ vs. the untreated group; ${ }^{* *} \mathrm{P}<0.01$ vs. the TNF- $\alpha$ onlytreated group.

with $\gamma$-tocotrienol reduced body fat mass in rats (15). A more recent study by Uto-Kondo et al showed that $\gamma$-tocotrienol suppressed adipocyte differentiation in 3T3-L1 preadipocytes (14). In the present study, we demonstrated for the first time that $\gamma$-tocotrienol effectively attenuated the TNF- $\alpha$-mediated increase in MCP-1 and IL-6 secretion and decrease in adiponectin secretion in 3T3-L1 adipocytes. Furthermore, the TNF- $\alpha$-induced changes in the mRNA expression of each adipokine were also inhibited by $\gamma$-tocotrienol. These results indicate that $\gamma$-tocotrienol affected the TNF- $\alpha$-mediated changes in the secretion of adipokines at the transcription level.

Activation of the transcription factor $N F-\kappa B$ is considered to play a major role in $\mathrm{TNF}-\alpha$-induced inflammatory responses, including down-regulation of adiponectin and up-regulation of MCP-1 and IL-6 in adipocytes (3-5). NF- $\kappa \mathrm{B}$ is activated by TNF- $\alpha$ via phosphorylation and removal of I $\mathrm{B}-\alpha$, resulting in its translocation to the nucleus and up-regulation of gene expression of pro-inflammatory adipokines, such as $M C P-1$ and $I L-6$ (3-5). Adiponectin suppression mediated by TNF- $\alpha$ is also regulated by $\mathrm{NF}-\kappa \mathrm{B}$ activation. Indeed, Kamon et al showed that TNF- $\alpha$-induced down-regulation of adiponectin secretion was cancelled by I $\kappa \mathrm{B}$ kinase $\beta$ inhibitor in 3T3-L1 adipocytes (20). Consistent with these studies, we observed that TNF- $\alpha$ enhanced the phosphorylation of I $\mathrm{B}$ - $\alpha$ and the nuclear translocation of $\mathrm{NF}-\kappa \mathrm{B}$.

Recent studies have demonstrated in different cell types that the anti-inflammatory effects of tocotrienols are mediated by suppression of the NF- $\mathrm{B}$ pathway (16). A tocotrienol-rich fraction of palm oil showed anti-inflammatory activity by inhibiting NF- $\kappa \mathrm{B}$ expression in human monocytic cells (17). Moreover, treatment of streptozotocin-induced diabetic rats with tocotrienols significantly suppressed the activation of the $\mathrm{NF}-\kappa \mathrm{B}$ pathway in the kidney and improved the renal function (21). In this study, our results show that $\gamma$-tocotrienol inhibits the TNF- $\alpha$-induced activation of NF- $\kappa \mathrm{B}$ in 3T3-L1 adipocytes. These observations indicate that $\gamma$-tocotrienol possesses antiinflammatory properties, such as attenuation of $M C P-1$ and IL-6 expression, through the suppression of NF- $\kappa \mathrm{B}$ activation in adipocytes. 
It is well known that adiponectin improves insulin sensitivity, partly through its anti-inflammatory effects $(9,22,23)$. Adiponectin is highly expressed in adipocytes, and is partly transcriptionally activated by $\operatorname{PPAR} \gamma$, which is negatively regulated by TNF- $\alpha$-induced inflammation (10). Previous studies have found that adiponectin treatment suppressed inflammation-mediated increase in MCP-1 and IL-6 production in 3T3-L1 cells, through attenuation of NF- $\kappa \mathrm{B}$ activation and increased PPAR $\gamma$ expression (23). Thus, MCP-1 and IL- 6 counteract adiponectin production in adipocytes. In our experiments, $\gamma$-tocotrienol reversed the TNF- $\alpha$-induced decrease in both adiponectin secretion and PPAR $\gamma$ expression. These results suggest that $\gamma$-tocotrienol regulates adiponectin production via PPAR $\gamma$, which may be involved in its antiinflammatory effects in adipocytes. Moreover, it has been demonstrated that the transcriptional activity of $N F-\kappa B$ is inhibited by PPAR $\gamma$ in other cell types (24). Thus, our results suggest that $\gamma$-tocotrienol down-regulates the activation of $\mathrm{NF}-\kappa \mathrm{B}$ in part by increasing PPAR $\gamma$ expression in adipocytes.

In summary, $\gamma$-tocotrienol inhibits the TNF- $\alpha$-induced inflammatory effects in 3T3-L1 adipocytes, and this action is mediated by suppression of $N F-\kappa B$ activation. These findings provide novel insight into the prevention and treatment of obesity-related pathologies.

\section{References}

1. Dandona P, Aljada A and Bandyopadhyay A: Inflammation: the link between insulin resistance, obesity and diabetes. Trends Immunol 25: 4-7, 2004.

2. Hotamisligil GS: Inflammation and metabolic disorders. Nature 444: 860-867, 2006.

3. Kim KY, Kim JK, Jeon JH, Yoon SR, Choi I and Yang Y: c-Jun $\mathrm{N}$-terminal kinase is involved in the suppression of adiponectin expression by TNF-alpha in 3T3-L1 adipocytes. Biochem Biophys Res Commun 327: 460-467, 2005.

4. Tak PP and Firestein GS: NF-kappaB: a key role in inflammatory diseases. J Clin Invest 107: 7-11, 2001.

5. Teferedegne B, Green MR, Guo Z and Boss JM: Mechanism of action of a distal NF-kappaB-dependent enhancer. Mol Cell Biol 26: 5759-5770, 2006.

6. Hotamisligil GS, Arner P, Caro JF, Atkinson RL and Spiegelman BM: Increased adipose tissue expression of tumor necrosis factor-alpha in human obesity and insulin resistance. J Clin Invest 95: 2409-2415, 1995.

7. Hotamisligil GS, Shargill NS and Spiegelman BM: Adipose expression of tumor necrosis factor-alpha: direct role in obesitylinked insulin resistance. Science 259: 87-91, 1993.
8. Cawthorn WP and Sethi JK: TNF-alpha and adipocyte biology. FEBS Lett 582: 117-131, 2008.

9. Maeda N, Takahashi M, Funahashi T, et al: PPARgamma ligands increase expression and plasma concentrations of adiponectin, an adipose-derived protein. Diabetes 50: 2094-2099, 2001.

10. Zhang B, Berger J, Hu E, et al: Negative regulation of peroxisome proliferator-activated receptor-gamma gene expression contributes to the antiadipogenic effects of tumor necrosis factor-alpha. Mol Endocrinol 10: 1457-1466, 1996.

11. Kamal-Eldin A and Appelqvist LA: The chemistry and antioxidant properties of tocopherols and tocotrienols. Lipids 31: 671-701, 1996.

12. Sen CK, Khanna S and Roy S: Tocotrienols in health and disease: the other half of the natural vitamin E family. Mol Aspects Med 28: 692-728, 2007.

13. Das S, Nesaretnam K and Das DK: Tocotrienols in cardioprotection. Vitam Horm 76: 419-433, 2007.

14. Uto-Kondo H, Ohmori R, Kiyose C, et al: Tocotrienol suppresses adipocyte differentiation and Akt phosphorylation in 3T3-L1 preadipocytes. J Nutr 139: 51-57, 2009.

15. Ima-Nirwana $S$ and Suhaniza $S$ : Effects of tocopherols and tocotrienols on body composition and bone calcium content in adrenalectomized rats replaced with dexamethasone. J Med Food 7: 45-51, 2004

16. Kaileh M and Sen R: Role of NF-kappaB in the anti-inflammatory effects of tocotrienols. J Am Coll Nutr 29: S334-S339, 2010

17. Wu SJ, Liu PL and Ng LT: Tocotrienol-rich fraction of palm oil exhibits anti-inflammatory property by suppressing the expression of inflammatory mediators in human monocytic cells. Mol Nutr Food Res 52: 921-929, 2008.

18. Ikeda S, Toyoshima K and Yamashita K: Dietary sesame seeds elevate alpha- and gamma-tocotrienol concentrations in skin and adipose tissue of rats fed the tocotrienol-rich fraction extracted from palm oil. J Nutr 131: 2892-2897, 2001.

19. Karin M, Yamamoto Y and Wang QM: The IKK NF-kappa B system: a treasure trove for drug development. Nat Rev Drug Discov 3: 17-26, 2004.

20. Kamon J, Yamauchi T, Muto S, et al: A novel IKKbeta inhibitor stimulates adiponectin levels and ameliorates obesity-linked insulin resistance. Biochem Biophys Res Commun 323: 242-248, 2004.

21. Kuhad A and Chopra K: Attenuation of diabetic nephropathy by tocotrienol: involvement of $\mathrm{NF}-\kappa \mathrm{B}$ signaling pathway. Life Sci 84: 296-301, 2009.

22. Yamauchi T, Kamon J, Waki H, et al: The fat-derived hormone adiponectin reverses insulin resistance associated with both lipoatrophy and obesity. Nat Med 7: 941-946, 2001.

23. Zoico E, Garbin U, Olioso D, et al: The effects of adiponectin on interleukin-6 and MCP-1 secretion in lipopolysaccharide-treated 3T3-L1 adipocytes: role of the NF-kappaB pathway. Int J Mol Med 24: 847-851, 2009.

24. Wang P, Anderson PO, Chen S, Paulsson KM, Sjogren HO and $\mathrm{Li}$ S: Inhibition of the transcription factors AP-1 and NF-kappaB in CD4 T cells by peroxisome proliferator-activated receptor gamma ligands. Int Immunopharmacol 1: 803-812, 2001. 